\title{
Recommendations of the Thoracic Imaging Section of the German Radiological Society for clinical application of chest imaging and structured CT reporting in the COVID-19 pandemic Empfehlungen der AG Thoraxdiagnostik der Deutschen Röntgengesellschaft zur klinischen Anwendung der Thoraxbildgebung und strukturierten CT-Befundung bei COVID-19-Pandemie
}

Authors

Jens Vogel-Claussen ${ }^{1,2}$, Julia Ley-Zaporozhan ${ }^{3,4}$, Prerana Agarwal ${ }^{5}$, Jürgen Biederer ${ }^{6,7,8,9}$, Hans-Ulrich Kauczor ${ }^{6,7}$, Sebastian Ley ${ }^{10,11}$, Hilmar Kühl ${ }^{12}$, Ullrich G. Mueller-Lisse $e^{3,4}$, Thorsten Persigehl' ${ }^{13}$, Christopher L. Schlett ${ }^{5}$, Dag Wormanns ${ }^{14,15}$, Gerald Antoch ${ }^{16 *}$, Okka W. Hamer ${ }^{17,18}$

Affiliations

1 Institut für Diagnostische und Interventionelle Radiologie, Medizinische Hochschule Hannover, Hannover, Deutschland

2 BREATH (Biomedical Research in End-stage and Obstructive Lung Disease Hannover), Deutsches Zentrum für Lungenforschung (DZL), Hannover, Deutschland

3 Klinik und Poliklinik für Radiologie, Ludwig-MaximiliansUniversität, München, Deutschland

4 Comprehensive Pneumology Center Munich (CPC-M), Deutsches Zentrum für Lungenforschung (DZL), München, Deutschland

5 Klinik für Diagnostische und Interventionelle Radiologie, Universitätsklinikum Freiburg, Medizinische Fakultät, Albert-Ludwigs-Universität Freiburg, Freiburg, Deutschland

6 Klinik für Diagnostische und Interventionelle Radiologie, Universitätsklinikum Heidelberg, Heidelberg, Deutschland

7 Translational Lung Research Center Heidelberg (TLRCH), Deutsches Zentrum für Lungenforschung (DZL), Heidelberg, Deutschland

8 Faculty of Medicine, University of Latvia, Riga, Lettland

9 Medizinische Fakultät, Christian-Albrechts-Universität zu Kiel, Kiel, Deutschland

10 Diagnostische und Interventionelle Radiologie, Chirurgisches Klinikum München-Süd, München, Deutschland

11 Klinik für Radiologie, Internistisches Klinikum MünchenSüd, München, Deutschland

12 Klinik für Radiologie, St.-Bernhard-Krankenhaus, Kamp-Lintfort, Deutschland

13 Institut für Diagnostische und Interventionelle Radiologie, Universitätsklinikum Köln, Köln, Deutschland

14 Radiologisches Institut, Evangelische Lungenklinik Berlin, Berlin, Deutschland

Präsident, Deutsche Röntgengesellschaft, Berlin, Deutschland.
15 Institut für Klinische Radiologie, Universitätsklinikum Münster, Münster, Deutschland

16 Institut für Diagnostische und Interventionelle Radiologie, Heinrich-Heine-Universität Düsseldorf, Düsseldorf, Deutschland

17 Institut für Röntgendiagnostik, Universitätsklinikum Regensburg, Regensburg, Deutschland

18 Abteilung für Radiologie, Lungenfachklinik Donaustauf, Donaustauf, Deutschland

Key words

lung, COVID-19, imaging, German Radiological Society

received 29.04.2020

accepted 05.05.2020

Bibliography

DOI https://doi.org/10.1055/a-1174-8378

Published online: 26.5.2020

Fortschr Röntgenstr 2020; 192: 633-640

(c) Georg Thieme Verlag KG, Stuttgart · New York

ISSN 1438-9029

Correspondence

Prof. Dr. med. Jens Vogel-Claussen

Vorsitzender der AG Thoraxdiagnostik der Deutschen

Röntgengesellschaft

Institut für Diagnostische und Interventionelle Radiologie Medizinische Hochschule Hannover, OE 8220,

Carl-Neuberg-Str. 1, 30625 Hannover

Vogel-Claussen.Jens@mh-hannover.de

\section{ABSTRACT}

This information provided by the Thoracic Imaging Section of the German Radiological Society is intended to give physicians recommendations on the use of thoracic imaging procedures in the context of the current COVID-19 pandemic. It represents the consensus of the authors based on the previous 
scientific knowledge and is intended to provide guidance for unified, structured CT reporting if COVID-19 pneumonia is suspected. The recommendations presented correspond to state of knowledge at the time of print and will be updated according to the results of ongoing and future scientific studies.

Key Points:

- COVID-19

- chest imaging

- German Radiological Society

\section{Citation Format}

- Vogel-Claussen J, Ley-Zaporozhan J, Agarwal P et al. Recommendations of the Thoracic Imaging Section of the German Radiological Society for clinical application of chest imaging and structured CT reporting in the COVID-19 pandemic. Fortschr Röntgenstr 2020; 192: 633640

\section{ZUSAMMENFASSUNG}

Diese Information der AG Thoraxdiagnostik der Deutschen Röntgengesellschaft soll Ärztinnen und Ärzten Empfehlungen zur Anwendung thoraxradiologischer Verfahren im Rahmen der aktuellen COVID-19-Pandemie geben. Sie repräsentiert den Konsensus der Autoren basierend auf den bisherigen wissenschaftlichen Erkenntnissen und soll zur einheitlichen und strukturierten CT-Befundung bei COVID-19 anleiten. Die vorgestellten Empfehlungen entsprechen dem Wissensstand zum Zeitpunkt der Drucklegung und werden entsprechend den Ergebnissen laufender und zukünftiger wissenschaftlicher Untersuchungen bei Bedarf aktualisiert.

\section{Introduction}

Severe acute respiratory syndrome coronavirus 2 (SARS-CoV-2) is spreading worldwide. Germany is also significantly affected by coronavirus disease 2019 (COVID-19) with currently over 157000 confirmed cases and 6115 deaths (as of April 29, 2020, Robert Koch Institute (RKI)).

At present, the only way to limit the spread of the virus is through far-reaching contact restrictions with drastic consequences for public life and the economy, thus early detection of those infected plays a decisive role in limiting the disease. Unfortunately, with a median incubation period of 5-6 days (1-14 days), carriers of the disease are already infectious before developing clinical symptoms. The definitive detection of SARS-CoV-2 is performed by polymerase chain reaction (PCR) of samples from the upper or lower respiratory tract [1]. However, the PCR may be negative in the early stages of the disease, as the sensitivity of the test depends on the local viral load, the quality of the sample and the location of the swab. False-negative test results promote further spread of the disease in the case of infection. In case of negative PCR and persistent clinical suspicion of COVID-19, serial PCR tests are therefore the method of choice [2]. However, experience from China shows that with a negative PCR test and typical clinical symptoms, a chest CT can show pneumonic lesions in the early stages of the disease which are suggestive of COVID-19 pneumonia [3, 4].

The role of imaging in COVID-19 is intensively discussed worldwide, especially since the body of data is developing dynamically with the duration and extent of the pandemic [3, 5-7].

This statement of the Thoracic Imaging Section of the German Radiological Society outlines the current scientific findings on imaging for COVID-19, and proposes a recommendation for imaging and uniform structured CT reporting for COVID-19. The preparation of radiology departments for COVID-19 and hygiene measures have already been published and are not the subject of this communication $[8,9]$.

\section{Current Scientific Background}

\section{Sensitivity and Specificity of Chest CT}

- Retrospective case series with 51 patients in China: In patients from endemic areas and with clinical symptoms, the PCR was initially negative in $29 \%$ of cases (15/51). In $98 \%$ of cases, CT was positive (50/51) and negative in one PCR-positive case [4].

- Retrospective case series with 1014 patients in China: In patients with negative PCR, the chest CT was positive in $75 \%$ (308/413) of cases. After analysis of serial PCR tests, the mean interval between initial negative and positive PCR results was $5.1 \pm 1.5$ days. The positive rates of the initial PCR test and chest CT imaging in this cohort were $59 \%(601 / 1014)$ and $88 \%(888 / 1014)$ for the diagnosis of patients with suspected COVID-19 [3].

- Retrospective case series with 158 patients in Italy: In $61 \%$ of cases $(96 / 158)$ the PCR was initially negative; in 42 of these 96 cases ( $44 \%$ ) the CT was positive. The sensitivity of the CT was $97 \%$, the specificity $56 \%$ [10].

- Retrospective case series with 81 patients in Wuhan: COVID-19 pneumonia was detectable in chest CT even in asymptomatic patients [11].

- Meta-analysis of COVID-19:

For the thoracic CT, the averaged sensitivity was $94 \%$ and the averaged specificity $37 \%$. The sensitivity of PCR was $89 \%$, thus test performance was strongly dependent on prevalence. In Germany with an assumed prevalence of $5.7 \%$, the calculated positive predictive value was $8.3 \%$ for CT and $84.3 \%$ for PCR; the negative predictive value was $99 \%$ for CT and $99.3 \%$ for PCR [12].

- Differentiation of COVID-19 from other viral pneumonia in CT: 
- Table 1 Categorizing CT changes during the COVID-19 pandemic based on recommendations of the Thoracic Imaging Section of the German Radiological Society.

category

1. CT changes suggestive of COVID-19 pneumonia (with high local prevalence and/or individual pre-test probability)

2. CT changes indeterminate, COVID-19 pneumonia possible

3. CT changes suggestive of an alternative diagnosis (e. g. bacterial pathogen spectrum)

4. no evidence of pneumonic opacities on $\mathrm{CT}$

\section{CT changes}

a. early dominant ground glass opacities

b. later dominant "crazy paving"/consolidation

c. signs of organizing pneumonia (e. g. arcade-like

consolidation/ground glass opacity, reversed halo sign)

d. peripheral and posterior predominance

e. round or geographically configured

f. bilateral, multifocal

g. intralesionally expanded vessels

h. absent mediastinal/hilar lymphadenopathy

a. ground glass/“crazy paving”/consolidation distributed differently than specified in Category 1 "CT changes suggestive of COVID-19"

b. central emphasis

e. non-round or non-geographically configured

a. nodules

b. "tree-in-bud"

c. peribronchial opacities

d. lobular/segmental consolidation

e. caverns

f. bronchial wall thickening

g. mucus plugging

h. pleural effusion

$-$

\section{radiological reporting template}

$\mathrm{CT}$ changes consistent with viral pneumonia with mild, moderate, severe extent. With high individual pretest probability suggestive of COVID-19 pneumonia. [Cov19Typ]

$\mathrm{CT}$ changes consistent with viral pneumonia with mild, moderate, severe extent. With high individual pretest probability COVID-19 pneumonia possible, but CT changes not characteristic. [Cov19Ind]

CT changes of lung parenchyma consistent with (alternative diagnosis). CT changes without indication of COVID-19 pneumonia. [Cov19Aty]

no evidence of pneumonic opacities on CT, thus currently no evidence of COVID-19 pneumonia. [Cov19Neg]
In a retrospective $\mathrm{CT}$ case series, three Chinese radiologists achieved sensitivities of $72 \%, 72 \%$ and $94 \%$ and specificities of $94 \%, 88 \%$ and $24 \%$ in distinguishing 219 cases of COVID-19 from 205 cases of non-COVID-19 viral pneumonia [13].

- Artificial intelligence support of radiology: In a retrospective $C T$ case series comprising patients with viral pneumonia ( $n=1186$, of which were COVID-19 positive $n=521)$, radiologists achieved a higher average accuracy (90\% vs. $85 \%, p<0.001)$, sensitivity ( $88 \%$ vs. $79 \%, p<0.001)$ and specificity ( $91 \%$ vs. $88 \%, p=0.001$ ) to distinguish COVID-19 from other pneumonia when the data were additionally evaluated using artificial intelligence [14].

\section{Potential Prognostic Value of Computed Tomography}

- Retrospective case series with 236 patients in Italy: After adjustment of patient demography and clinical parameters, a well-ventilated lung parenchyma of less than $73 \%$ in CT at hospital admission was significantly associated with later admission to intensive care or death (OR 5.4, p<0.001) [14].

\section{Morphology of COVID-19 Pneumonia on Chest CT}

Taking into account the available literature [7, 11, 15-18], it appears useful for further clinical management to divide patients with symptoms of an acute respiratory infection during the pandemic into the following 4 groups based on CT morphology ( Table 1):

\section{CT groups}

1. Suggestive of COVID-19 pneumonia

2. Indeterminate: corresponds to viral pneumonia; COVID-19 possible during high prevalence

3. Suggestive of alternative diagnosis

4. No evidence of pneumonic opacities

\section{Group 1: CT changes suggestive of COVID-19 pneumonia}

COVID-19 pneumonia most commonly manifests itself in the form of ground glass opacities, followed by a mixed picture of ground glass opacities and consolidations. Likewise, crazy paving (ground glass opacity overlaid by smoothly bordered reticulations) can be present, especially in late stages of the disease. A positive air bronchogram is typical. Numerous studies describe dilation of the pulmonary vessels within the lesion or perilesionally. In some cases the halo sign (central consolidation with surrounding ground glass) and the reversed halo sign (central ground glass with surrounding consolidation) were observed. The lesions are bilateral and multifocal. They favor the middle and lower lungs and periphery of the lungs, especially the posterior portions. The lesions are patchy, round or geographically shaped. A band-like configuration may predominate in the recovery phase ( $\triangleright$ Fig. 1).

\section{Group 2: CT changes indeterminate, COVID-19 is possible}

These patients also have ground glass opacities, crazy paving, consolidations or a mixed picture. The borders, configuration 


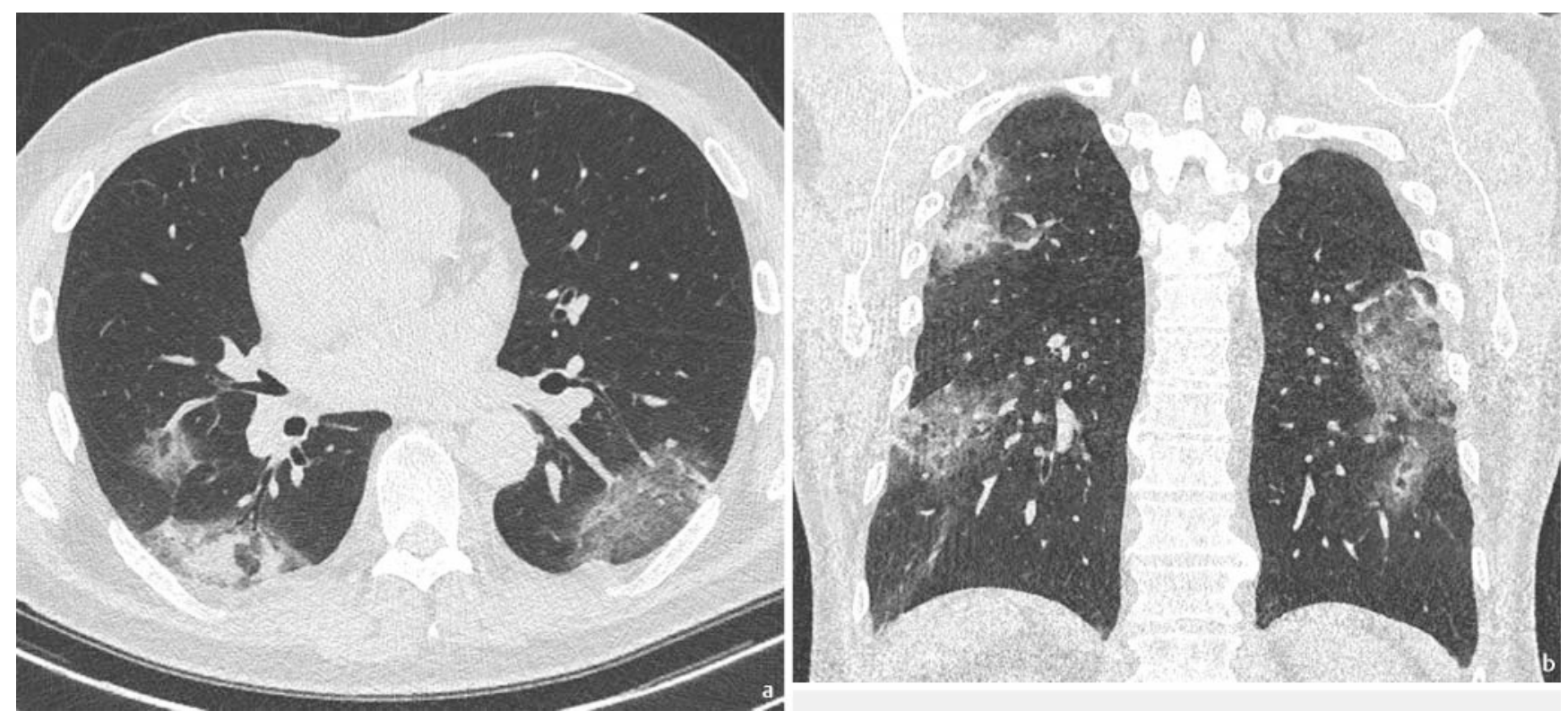

- Fig. 1 CT changes suggestive of COVID-19 pneumonia Computed tomography of a 61-year-old male patient with PCR-confirmed SARS-CoV2 infection exhibits several peripheral and dorsally predominant bilateral consolidations and ground glass-opacities in the lung (axial a and coronal plane b).
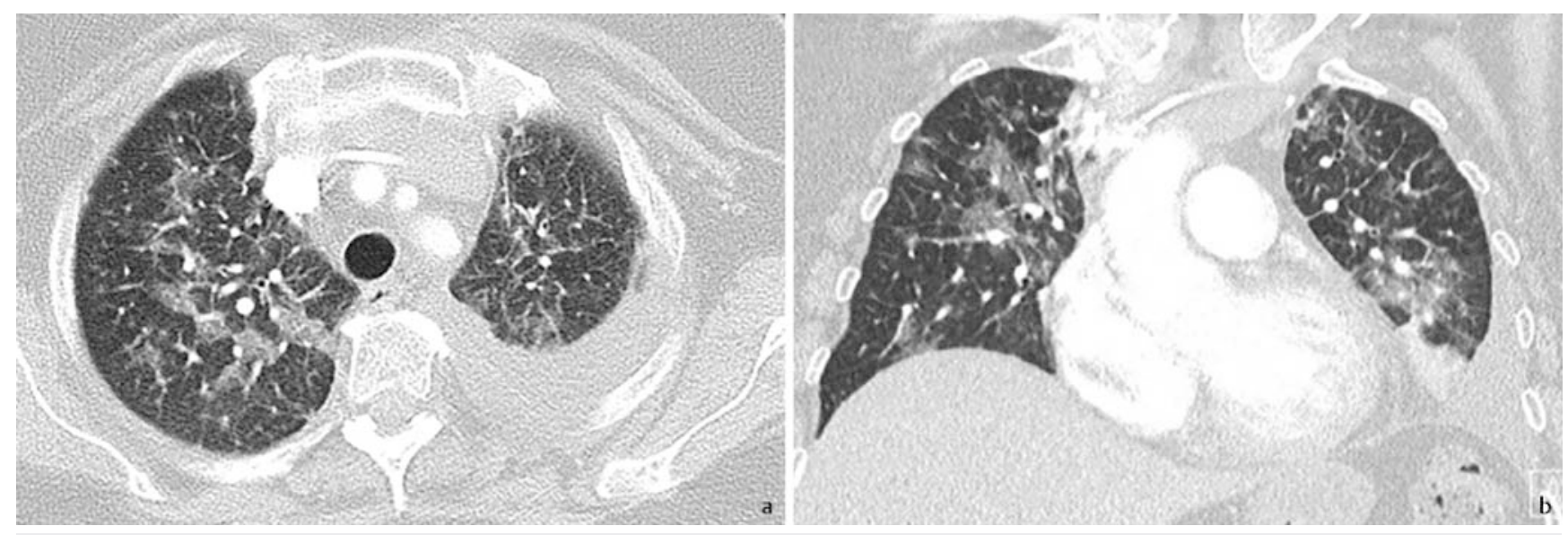

- Fig. 2 CT changes indeterminate, COVID-19 is possible. 55-year-old female patient with bronchial carcinoma of the left inferior lobe (not shown) and pleural effusion on the left side in known pleural carcinosis. The patient presented herself to the emergency room due to a malaise that had existed for one day. Laboratory chemistry showed increased D-dimers, which led to the suspicion of a pulmonary embolism. Contrast-enhanced computed tomography shows bilateral centrally accentuated ground glass opacity and slight thickening of the interlobular septa (axial a and coronal plane b). Pulmonary embolism was not apparent. RT-PCR confirmed COVID-19.

and distribution of the lesions do not meet the criteria of the Group 1 patients. If the clinical symptoms are appropriate, this CT image primarily suggests a viral pneumonia, although no statement can be made about the pathogen. COVID-19 pneumonia is possible ( $\vee$ Fig. 2,3 ).

\section{Group 3: CT changes suggestive of an alternative diagnosis}

Patients in Group 3 have indications that have never or only rarely been described in COVID-19 pneumonia, including nodules, "tree-in-bud" sign, caverns, bronchial wall thickening and mucus plugging. Pleural effusions or lymphadenopathy rarely occur.
With related symptoms, a differential diagnosis can be pneumonia due to another pathogen, but also a superinfection ( $>$ Fig.4).

\section{The Role of Imaging during the COVID-19 Pandemic}

The findings in COVID-19 on chest radiography and CT are not specific and can also occur in other viral pneumonia [19].

In the context of the COVID-19 pandemic, CT and chest radiography make a decisive contribution to the clinical management of the disease: the evaluation of the initial extent of the disease, 


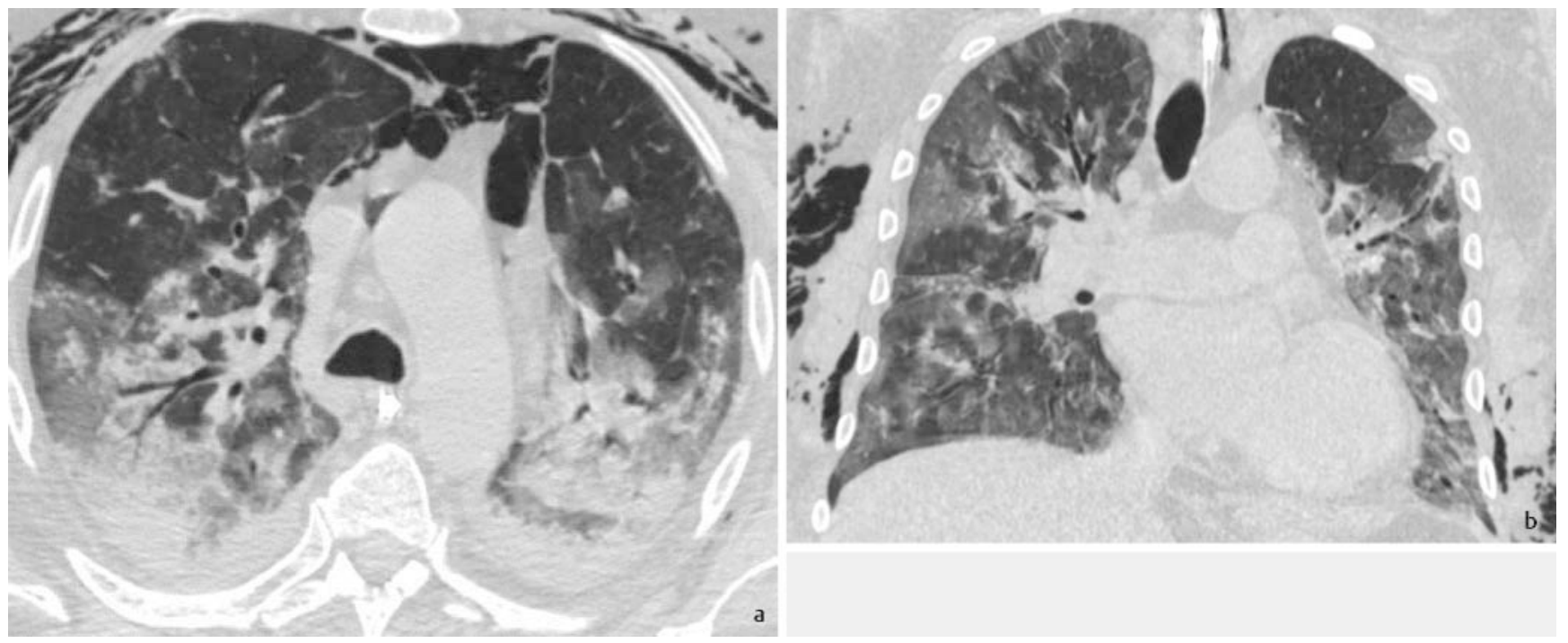

- Fig. 3 CT changes indeterminate, COVID-19 is possible. 56-year-old male patient intubated and ventilated due to clinical ARDS (Acute Respiratory Distress Syndrome) and pneumonia of unknown origin. The chest CT shows dorsally pronounced, central and peripherally extended consolidations and ground glass-opacities as well as incidental pneumomediastinum and air inclusions in the thoracic wall (axial a and coronal plane b). Laboratory chemistry revealed influenza A virus H1N1 pneumonia.
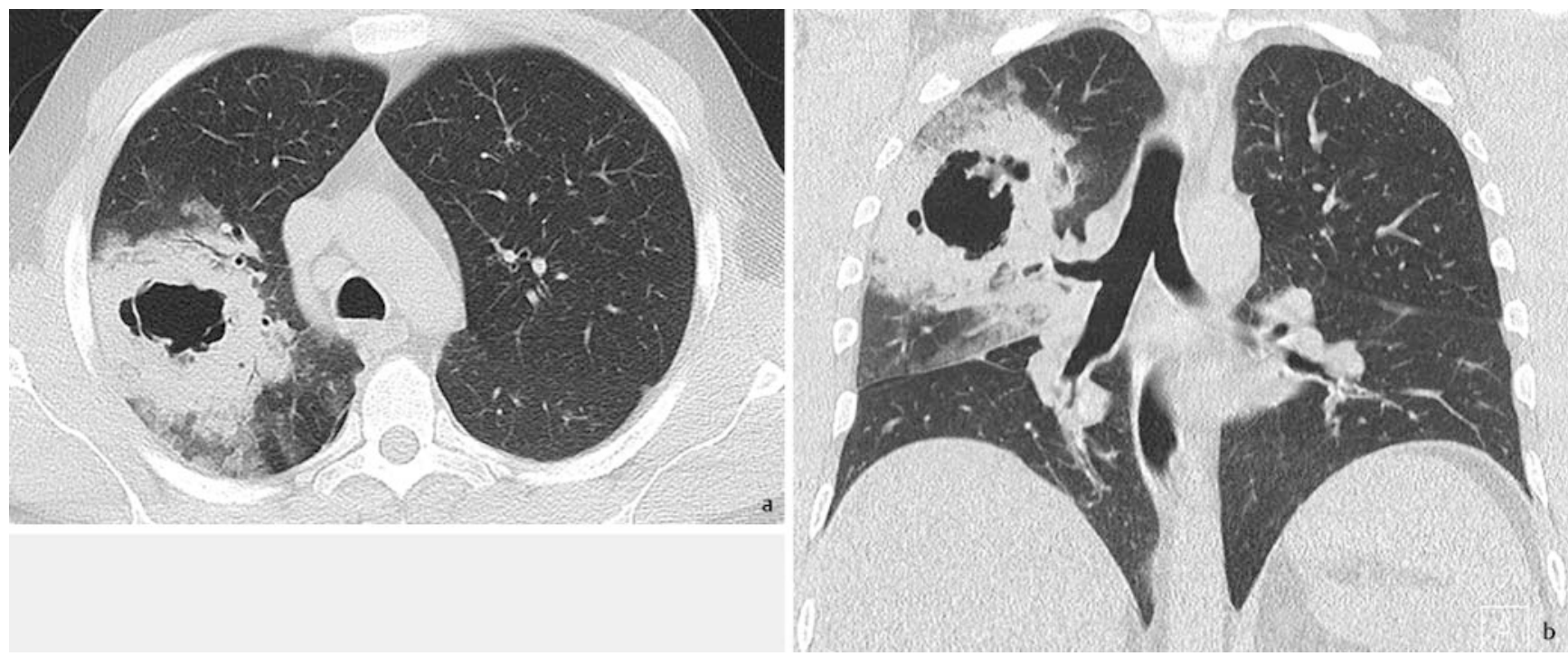

- Fig. 4 CT changes suggestive of an alternative diagnosis (e. g. bacterial pathogen spectrum). Computed tomography of a 41-year-old male patient shows consolidation in the right superior lobe with central cavitation with streptococcus pyogenes pneumonia (axial a and coronal plane b). PCR for COVID-19 negative.

the diagnosis of pneumonia-associated complications and the progression assessment in severe cases. CT can contribute to the interdisciplinary evaluation of individual prognosis. Chest radiography and CT are therefore typically used not as a screening test but for the clinical evaluation of patients with respiratory symptoms such as dyspnea and oxygen desaturation. The indication is not only based on the severity of the disease but also on the clinical relevance for the further management of the individual patient.

For patients in intensive care units, a mobile radiograph can be taken to assess the severity of the illness and to monitor the course of the disease if clinically indicated. Daily monitoring with chest radiography is not recommended [6]. For intensive care patients with COVID-19 and clinical indication, an ultrasound examination can also be helpful in assessing the course of the disease, e. g. pleural effusions, consolidation or atelectasis [20].

Taking into account the current state of knowledge, imaging within the framework of the COVID-19 pandemic fulfills 5 core tasks: 


\section{(2) AC Thoraxdiagnostik \\ Structured non-contrast CT report in patients with suspected COVID-19 \\ Indication: \\ (A justifying clinical indication is mandatory) \\ Findings: \\ No prior examination available for comparison \\ Comparison with CT of: \\ Lung parenchyma / airways: \\ Opacification: none / ground glass / crazy paving / consolidation / tree-in-bud / reversed halo sign / interstitial pulmonary edema / non-specific pattern \\ Pulmonary lobes: upper lobe / middle lobe / lower lobe, left / right / bilateral \\ Dominant distribution pattern: peripheral / central / peribronchial \\ Extent: mild / moderate / severe \\ Compared to prior examination: increased / decreased / completely gone / unchanged \\ Emphysema: none / mild / moderate / severe \\ Fibrosis: none / mild / moderate / severe}

Pleura: pleural effusion left / right / bilateral / mild / moderate / severe

Lymph nodes: no lymphadenopathy / mediastinal lymphadenopathy

Mediastinum:

Heart: normal / enlarged (specifically RA, LA, RV, LV) / dilation of main

stem of pulmonary artery / aneurysm

Coronary artery calcifications: none / mild / moderate / severe

Pericardial effusion: yes / no

MSK: normal / degenerative changes

Miscellaneous:

\section{$-\mathrm{C}$}

ACThoraxdiagnostik

AC Thoraxdiagnostik

Impression:

1. Diagnosis and extent

CT changes consistent with viral pneumonia with mild / moderate / severe extent. With high individual pretest probability suggestive of COVID-19 pneumonia. [Cov19Typ]

CT changes consistent with viral pneumonia with mild / moderate / severe extent. With high individual pretest probability COVID-19 pneumonia possible, but CT changes not characteristic. [cov191nd]

CT changes of lung parenchyma consistent with (alternative diagnosis). CT changes without suspicion of COVID-19 pneumonia. [Cov19Aty]

No evidence of pneumonic opacities in CT, thus currently no evidence of COVID-19 pneumonia. [Cov19Neg]

2. Comparison to prior exam:

Lung opacities on CT are increased / decreased / completely gone / unchanged. Currently additional superinfection possible.

3. Relevant additional findings:

Pulmonary emphysema, pulmonary fibrosis, pleural effusion, atherosclerosis, aneurysm, etc.

Structured Reporting Template of the Thoracic Imaging Section of the German Radiological Society https://www.ag-thorax.drg.de/deDE/6284/covid-19/

[Cov19] Coding according to RSNA recommendations: https://doi.org/10.1148/ryct.2020200152

Fig. 5 Structured reporting based on native CT in patients with suspected COVID-19 according to the recommendation of the Thoracic Imaging Section of the German Radiological Society.

\section{Support of diagnosis}

As noted above, CT changes are not specific for the diagnosis of COVID-19 pneumonia, but can be suggestive.

Based on CT changes suggestive of COVID-19 pneumonia, a suspected diagnosis can be made in the presence of: 1 . appropriate clinical symptoms, 2 . negative PCR test, 3 . high individual pretest probability with high local prevalence of SARS-Cov-2, and 4. clinical indication in patients with pronounced symptoms requiring hospitalization. However, the diagnosis has to be confirmed by a series of PCR tests. A negative CT-based diagnosis does not rule out COVID-19. Initial results demonstrating that artificial intelligence supports the radiologist in making a tentative diagnosis are promising, but they need to be further developed and tested in larger prospective multicenter studies.

\section{Assessment of the severity of lung parenchyma changes and monitoring}

CT allows a reliable assessment of the initial extent of the disease. $\mathrm{CT}$ and chest radiography allow an assessment of the course of the disease to support clinical evaluation. Imaging can thus contribute to an interdisciplinary analysis to assess the individual prognosis of patients.

\section{Detection of complications}

Chest radiography and CT in particular can detect complications. In addition to superinfection, thrombotic and embolic events should be particularly mentioned, since COVID-19 can obviously lead to hypercoagulopathy $[21,22]$. Such complications should be treated with intravenous contrast, and a low-dose CT strategy should be abandoned. 


\section{Detection of "incidental" COVID-19 pneumonia}

Findings suggestive of COVID-19 pneumonia found in a thoracic $\mathrm{CT}$ performed for another indication must be recognized by the radiologist and immediately reported to the referring physician. The diagnosis has to be then confirmed by a PCR test.

\section{Triage in an environment with limited resources}

In a publication of the international Fleischner Society describing the use of imaging in different clinical scenarios, imaging is also discussed as a triage procedure when high case numbers and limited availability of virological tests leave no other choice [6]. However, such a scenario seems rather unlikely in Germany at present, given declining infection numbers and sufficient PCR test capacity.

\section{Structured CT Reporting when COVID-19 is suspected}

CT examinations in suspected cases of COVID-19 should be performed as non-contrast thin-slice CT with a low-dose protocol, unless further differential diagnoses indicate the administration of contrast. The radiological findings should be categorized into four groups as follows ( $\triangleright$ Table 1 ):

1. CT changes suggestive of COVID-19 pneumonia (with high local prevalence and/or individual pre-test probability) [Cov19typ]

2. CT changes indeterminate, COVID-19 pneumonia is possible [Cov19Ind]

3. CT changes suggestive of an alternative diagnosis

(e.g. bacterial pathogen spectrum) [Cov19Aty]

4. No indication of pneumonic lesions on CT [Cov19Neg]

This classification as well as the corresponding formulations in the structured reporting template ( $\boldsymbol{\nabla}$ Fig. 5 ) were prepared by the Board of the Thoracic Imaging Section of the German Radiological Society in cooperation with colleagues from the University Hospitals of Freiburg and Cologne, and according to expert opinion, best reflect the validity of CT when COVID-19 is suspected. It is largely congruent with the recommendations of the RSNA [7]. Only in category 3 was an alternative CT diagnosis emphasized and not the "atypical COVID 19 pattern", in order to make it clear to the referring physicians that the radiologist sees a different diagnosis here than COVID-19 pneumonia (e.g. bacterial lobar pneumonia).

The image data collected worldwide as a result of the pandemic is being collected and networked both nationally and internationally in various databases in order to gain important new knowledge needed for COVID-19 but also for possible future virus pandemics. In order to facilitate data searches and the networking of image data with the corresponding findings internationally, the [Cov19] coding according to RSNA recommendations was integrated into the assessment of the structured reporting template [7]. The assessment of the extent of pneumonic lesions appears to be prognostically important. However, a mandatory quantitative evaluation in clinical routine was not carried out, as the added value is currently not scientifically proven [23]. In addition to the evaluation of pneumonia, the structured reporting of e. g. cardiopulmonary comorbidities on the non-contrast chest CT is important, which is helpful for the clinical interdisciplinary evaluation of the patient.

\section{Summary and Outlook}

These recommendations of the Thoracic Imaging Section ofthe German Radiological Society are based on the current state of knowledge at the time of writing on 29 April 2020 and are intended to provide physicians with guidance on the use of chest imaging in the current COVID-19 pandemic. It represents the current status in a highly dynamic environment and is likely to undergo repeated adjustments in the further course of time.

\section{Conflict of Interest}

The authors declare that they have no conflict of interest.

\section{References}

[1] RKI. Im Internet: https://www.rki.de/DE/Content/InfAZ/N/Neuartiges_C

[2] RKI. Im Internet: https://www.rki.de/DE/Content/InfAZ/N/Neuartiges_ Coronavirus/Vorl_Testung_nCoV.html

[3] Ai T, Yang Z, Hou $\mathrm{H}$ et al. Correlation of Chest CT and RT-PCR Testing in Coronavirus Disease 2019 (COVID-19) in China: A Report of 1014 Cases. Radiology 2020. doi:10.1148/radiol.2020200642

[4] Fang Y, Zhang H, Xie J et al. Sensitivity of Chest CT for COVID-19: Comparison to RT-PCR. Radiology 2020. doi:10.1148/ radiol.2020200432

[5] Sverzellati N, Milone F, Balbi M. How imaging should properly be used in COVID-19 outbreak: an Italian experience. Diagnostic Interv Radiol 2020. doi:10.5152/dir.2020.30320

[6] Rubin GD, Haramati LB, Kanne JP et al. The Role of Chest Imaging in Patient Management during the COVID-19 Pandemic: A Multinational Consensus Statement from the Fleischner Society. Radiology 2020. doi:10.1148/radiol.2020201365

[7] Simpson S, Kay FU, Abbara S et al. Radiological Society of North America Expert Consensus Statement on Reporting Chest CT Findings Related to COVID-19. Endorsed by the Society of Thoracic Radiology, the American College of Radiology, and RSNA. Radiol Cardiothorac Imaging 2020. doi:10.1148/ryct.2020200152

[8] Antoch G, Urbach H, Mentzel H] et al. SARS-CoV-2/COVID-19: Empfehlungen für die Radiologische Versorgung - Eine Stellungnahme, der Deutschen Röntgengesellschaft (DRG), der Deutschen Gesellschaft für Neuroradiologie (DGNR), der Gesellschaft für Pädiatrische Radiologie (GPR), der Deutschen Gese. Rofo 2020; 192: 418-421. doi:10.1055/ a-1149-3625

[9] Mossa-Basha M, Meltzer CC, Kim DC et al. Radiology Department Preparedness for COVID-19: Radiology Scientific Expert Panel. Radiology 2020. doi:10.1148/radiol.2020200988

[10] Caruso D, Zerunian M, Polici M et al. Chest CT Features of COVID-19 in Rome, Italy. Radiology 2020. doi:10.1148/radiol.2020201237

[11] Shi $H$, Han X, jiang $N$ et al. Radiological findings from 81 patients with COVID-19 pneumonia in Wuhan, China: a descriptive study. Lancet Infect Dis 2020; 20: 425-434 
[12] Kim H, Hong H, Yoon SH. Diagnostic Performance of CT and Reverse Transcriptase-Polymerase Chain Reaction for Coronavirus Disease 2019: A Meta-Analysis. Radiology 2020. doi:10.1148/radiol.2020201343

[13] Bai HX, Hsieh B, Xiong Z et al. Performance of radiologists in differentiating COVID-19 from viral pneumonia on chest CT. Radiology 2020. doi:10.1148/radiol.2020200823

[14] Bai HX, Wang R, Xiong Z et al. Al Augmentation of Radiologist Performance in Distinguishing COVID-19 from Pneumonia of Other Etiology on Chest CT. Radiology 2020; 201491: doi:10.1148/radiol.2020201491

[15] Inui S, Fujikawa A, Jitsu $M$ et al. Chest CT Findings in Cases from the Cruise Ship “Diamond Princess” with Coronavirus Disease 2019 (COVID-19). Radiol Cardiothorac Imaging 2020. doi:10.1148/ ryct. 2020200110

[16] Song F, Shi N, Shan F et al. Emerging 2019 novel coronavirus (2019-NCoV) pneumonia. Radiology 2020. doi:10.1148/ radiol.2020200274

[17] Ng MY, Lee EY, Yang J et al. Imaging Profile of the COVID-19 Infection: Radiologic Findings and Literature Review. Radiol Cardiothorac Imaging 2020. doi:10.1148/ryct.2020200034
[18] Kanne JP, Little BP, Chung JH et al. Essentials for Radiologists on COVID-19: An Update - Radiology Scientific Expert Panel. Radiology 2020. doi:10.1148/radiol.2020200527

[19] Koo HJ, Lim S, Choe J et al. Radiographic and CT features of viral pneumonia. Radiographics 2018; 38: 719-739

[20] Sofia S, Boccatonda A, Montanari M et al. Thoracic ultrasound and SARS-COVID-19: a pictorial essay. J Ultrasound 2020. doi:10.1007/ s40477-020-00458-7

[21] Songping C, Shuo C, Xiunan L et al. Prevalence of venous thromboembolism in patients with severe novel coronavirus pneumonia. J Thromb Haemost 2020. doi:10.1111/jth.14830

[22] Klok FA, Kruip MJHA, van der Meer NM et al. Incidence of thrombotic complications in critically ill ICU patients with COVID-19. Thromb Res 2020. doi:10.1016/j.thromres.2020.04.013

[23] Colombi D, Bodini FC, Petrini M et al. Well-aerated Lung on Admitting Chest CT to Predict Adverse Outcome in COVID-19 Pneumonia. Radiology 2020; 201433: doi:10.1148/radiol.2020201433 